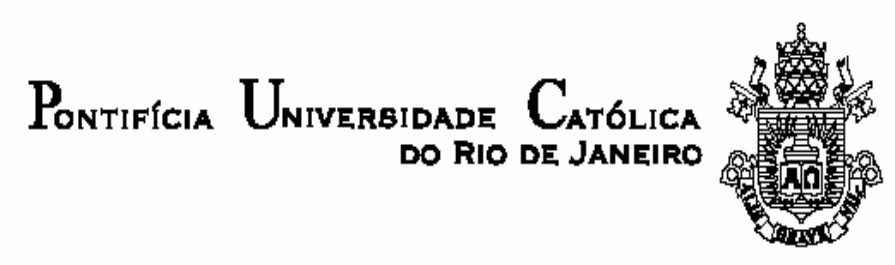

Michelle Borely Ferreira de Lima

Os desafios do Assistente Social no trabalho com crianças e adolescentes em situação de rua atendidos em uma Central de Recepção no município do Rio de Janeiro

Dissertação de Mestrado

Dissertação apresentada como requisito parcial para a obtenção de grau de Mestre pelo Programa de PósGraduação em Serviço Social do Departamento de Serviço Social da PUC-Rio.

Orientadora: Professora Irene Rizzini

Rio de Janeiro

Outubro de 2008 


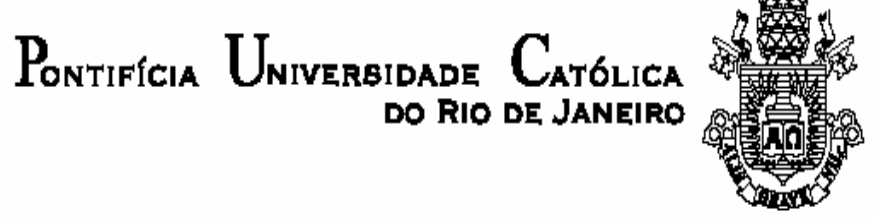

Michelle Borely Ferreira de Lima

\section{Os desafios do Assistente Social no trabalho com crianças e adolescentes em situação de rua atendidos em uma Central de Recepção no município do Rio de Janeiro}

Dissertação de Mestrado

Dissertação apresentada como requisito parcial para a obtenção de grau de Mestre pelo Programa de Pós-Graduação em Serviço Social do Departamento de Serviço Social do Centro de Ciências Sociais da PUC-Rio. Aprovada pela Comissão Examinadora abaixo assinada.

Prof. Irene Rizzini

Orientadora

Departamento de Serviço Social da PUC-Rio

Prof. Sueli Bulhões da Silva

Departamento de Serviço Social da PUC-Rio

Prof. Luciene Naiff

Programa de Pós-graduação em Psicologia da UNIVERSO

Prof. Nizar Messari

Vice-Decano de Pós-Graduação do Centro de Ciências Sociais - PUC-Rio

Rio de Janeiro, 29 de outubro de 2008 
Todos os direitos reservados. É proibida a reprodução total ou parcial do trabalho sem autorização da universidade, da autora do orientador.

\section{Michelle Borely Ferreira de Lima}

Graduou-se em Serviço Social na Universidade Federal do Rio de Janeiro em 2002. Especialista em Saúde da Família pela Universidade Federal Fluminense em 2005. Assistente Social da Prefeitura do Rio de Janeiro desde 2003.

Ficha Catalográfica

Lima, Michelle Borely Ferreira de

Os desafios do assistente social no trabalho com crianças e adolescentes em situação de rua atendidos em uma Central de Recepção no município do Rio de Janeiro / Michelle Borely Ferreira de Lima ; orientadora: Irene Rizzini. 2008.

120 f. : II. ; $30 \mathrm{~cm}$

Dissertação (Mestrado em Serviço Social)Pontifícia Universidade Católica do Rio de Janeiro, Rio de Janeiro, 2008.

Inclui bibliografia

1. Serviço Social - Teses. 2. Crianças e adolescentes em situação de rua. 3. Assistentes sociais. 4. Desafios. 5. Política Nacional de Assistência Social. I. Rizzini, Irene. II. Pontifícia Universidade Católica do Rio de Janeiro. Departamento de Serviço Social. III. Título.

CDD: 361 


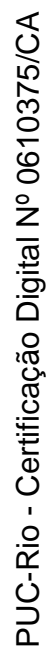

Aos meus pais Lêda \& Carlos o meu Muito Obrigado! 


\section{Agradecimentos}

Agradeço em primeiro lugar aos meus pais: Lêda Borely Ferreira de Lima e Carlos Roberto de Lima, que me propiciaram chegar aonde eu cheguei.

Agradeço ao meu marido Jader Leandro Silva Ferrari, pelo o seu apoio incondicional.

Agradeço ao meu filho do coração Leandro Gabriel Ferrari e aos que estão ainda por vir. Pois é pensando no futuro deles que estou aqui hoje.

Agradeço a minha orientadora Irene Rizzini, pela a sua dedicação e incentivo. Por acreditar mesmo quando eu não acreditava.

Agradeço aos amigos da pesquisa "Do confinamento ao acolhimento", da qual fiz parte como pesquisadora, pelo o apoio, ensinamentos e pelas ótimas manhãs de sexta-feira.

Agradeço a amiga e professora Luciene Naiff, pela disponibilidade nos momentos difíceis e por lembrar que só temos essa idade uma vez na vida, que o tempo não volta e que é preciso, às vezes, rever as prioridades.

Agradeço a professora Sueli Bulhões, pela contribuição na defesa desta dissertação.

Agradeço a professora Nívia Valença, por ensinar a olhar as outras possibilidades e me apresentar a PUC-Rio.

Agradeço a professora Ludmila Fontenele Cavalcanti, pelo o incentivo de sempre continuar...

Agradeço a PUC-Rio pela a bolsa FRAC, pois sem ela esse caminho seria inviável.

Agradeço a minha prima Mary Luce Ferreira Conz, por acreditar deste o início que seria possível.

Agradeço a minha tia e madrinha Lisete Ferreira Conz pelo o cuidado e carinho.

Agradeço a todos os professores e amigos que de uma maneira ou de outra contribuíram para a realização deste trabalho.

Obrigada a todos!

Michelle Borely Ferreira de Lima 


\section{Resumo}

Borely, Michelle; Rizzini, Irene. Os desafios do Assistente Social no trabalho com crianças e adolescentes em situação de rua atendidos em uma Central de Recepção no município do Rio de Janeiro. Rio de Janeiro, 2008, 120p. Dissertação de Mestrado, Departamento de Serviço Social, Pontifícia Universidade Católica do Rio de Janeiro.

Esta dissertação tem por objetivo estudar os desafios de assistentes sociais que trabalham em uma Central de Recepção no município do Rio de Janeiro, voltado para o atendimento a crianças e adolescentes em situação de rua. Partimos da observação, em nosso cotidiano de trabalho, de que há um "novo perfil” de crianças e adolescentes em situação de rua. Ao longo dos últimos anos, temos constatado o crescente envolvimento desta população com o mundo das drogas, seja como usuários ou como parte do tráfico de drogas. Verificamos que este envolvimento, com freqüência, os leva à expulsão da comunidade onde residem. Destacamos ainda, como fenômenos atuais, o advento do uso do crack e a presença de um número maior de meninas em situação de rua. Como metodologia, utilizamos uma abordagem qualitativa, ilustrando nossa discussão com entrevistas realizadas com assistentes sociais e a diretora do Central de Recepção. Os resultados foram agrupados em quatro categorias, que são: (1) Visões das assistentes sociais sobre as políticas públicas e serviços oferecidos; (2) Questão da abordagem; (3) Falta de retaguarda e (4) Precariedade de infraestrutura no atendimento a este grupo; (4) O mundo das drogas: a grande incógnita?

\section{Palavras-chave}

Crianças e adolescentes em situação de rua; assistentes sociais; desafios; Política Nacional de Assistência Social. 


\section{Abstract}

Borely, Michelle; Rizzini, Irene. Social Workers' challenges in the work with street children and youth in a Reception Center in Rio de Janeiro. Rio de Janeiro, 2008, 120p. MSc. Dissertation, Departamento de Serviço Social, Pontifícia Universidade Católica do Rio de Janeiro.

This dissertation aims to study the challenges of Social Service Agents who work in a Reception Center in the municipality of Rio de Janeiro. This Center is responsible for the reception and the referral of children and youth who were caught on the streets on their own. Based on our own experience, we start with the assumption that there is a "new profile" of children in the situation of the streets. In the last few years, we have observed that many of them have developed a growing involvement with the world of drugs, both as consumers and as participants of the drug trade. Many of them end up being forced out of their homes. In Addition, we would like to highlight two other aspects: the increasing presence of girls among the street population and the growing use of Crack by this group. Regarding the methodology, we used a qualitative approach and illustrated our discussion with interviews conducted with social workers and the director of the Reception Center. The results were grouped in 5 categories: (1) The social workers views about public policies and social services ; (2) Outreach work on the streets; (3) Lack of support; (4) Precariousness of infrastructure in services aimed at this group; (5) The world of drugs: the big enigma?

\section{Keywords}

Children and adolescents in the situation of the streets; social workers; challenges; National Social Welfare Policy. 


\section{Sumário}

1. Introdução 12

1.1. Estrutura da dissertação 20

2. A Questão da Criança e Adolescente em situação de Rua na Literatura

2.2. Uma reflexão sobre a questão da exclusão

3. A distância entre teoria e prática: a retórica dos direitos e 0 que acontece na prática

3.1. O Sistema Único de Assistência Social (SUAS).

4. Os desafios dos assistentes sociais que trabalham com crianças e adolescentes em situação de rua numa Central de Recepção 
4.6. Visões das Assistentes Sociais sobre as Políticas Públicas

e serviços oferecidos

4.7. A questão da Abordagem

4.8. Falta de retaguarda

76

4.9. Falta de intra-estrutura

78

4.10. O novo perfil dos/as meninos/as em situação de rua e o mundo das drogas. O mundo das drogas: a grande incógnita?

5. Considerações finais

6. Referências Bibliográficas

Anexo 1

93

Relatório de identificação do perfil da população usuária e organização do sistema de abrigamento no período de janeiro a maio de 2007

Anexo 2

Diretrizes Normativas e Operacionais para o trabalho das Centrais de Recepção de Crianças e Adolescentes em situação de rua 


\section{Lista de Gráficos:}

Gráfico 1- Atendimentos X Evasões na Central de Recepção

Gráfico 2- O que o fez vir para as ruas?

Gráfico 3 - Encaminhamento para abrigos da rede própria e conveniada

Gráfico 4 - Levantamento por faixa etária

Gráfico 5 - Fluxo de Atendimentos

Gráfico 6 - Sexo X Idade dos usuários da Central de Recepção 


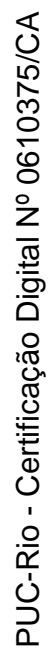

"Todo ponto de vista é a vista de um ponto".

Frei Betto 